\title{
Perovskite fever
}

\author{
Staggering increases in the performance of organic-inorganic perovskite solar cells have renewed \\ the interest in these materials. However, further developments and the support from academic and \\ industrial partners will hinge on the reporting of accurate efficiency values.
}

It is difficult to resist the intense appeal of hybrid organic-inorganic perovskites. At least, this is what the recent large increase in the number of presentations on perovskite solar cells at international conferences and of published articles on the topic seems to suggest. The performance of these materials in photovoltaic applications, which in a few years has surpassed that of all alternative technologies based on solution-processed materials, is, in fact, exceptionally promising.

Crystal structures of hybrid perovskites have been known for more than a century. Their optoelectronic characteristics make them attractive for the realization of fieldeffect transistors, bright-emitting sources and lasers ${ }^{1}$. At present, however, most of the attention from researchers is focused on photovoltaic applications. In a Commentary on page 838, Michael Grätzel discusses in detail the properties, device embodiments and deposition techniques that led to the latest power-conversion efficiencies of perovskite solar cells. On page 897, Sang Il Seok and colleagues describe a solvent-engineering strategy that boosts the power-conversion efficiency up to a certified value of $16.2 \%$. Improving this approach, the same research group has already certified solar cells with an efficiency of $17.9 \%{ }^{2}$. In a related News \& Views on page 845, Michael McGehee argues that limited light-conversion performance is unlikely to hamper the industrial deployment of this technology. Rather, some unique complexities of hybrid perovskite solar cells, such as their poor stability in humid air and the risk that these devices may release lead - an element that is key to reach the best performance reported so far - to the environment, have raised concerns that will have to be addressed before the cells can be commercialized.

These issues notwithstanding, the technological transfer of perovskite photovoltaics is already in progress. Oxford Photovoltaics, a spin-out company from the University of Oxford, is leveraging this technology to develop cheap semitransparent light-harvesting coatings to be integrated in architectural glass ${ }^{3}$. As with the coloured dye-sensitized solar windows recently installed in the SwissTech Convention Centre of the École Polytechnique Fédérale de Lausanne ${ }^{4}$, other

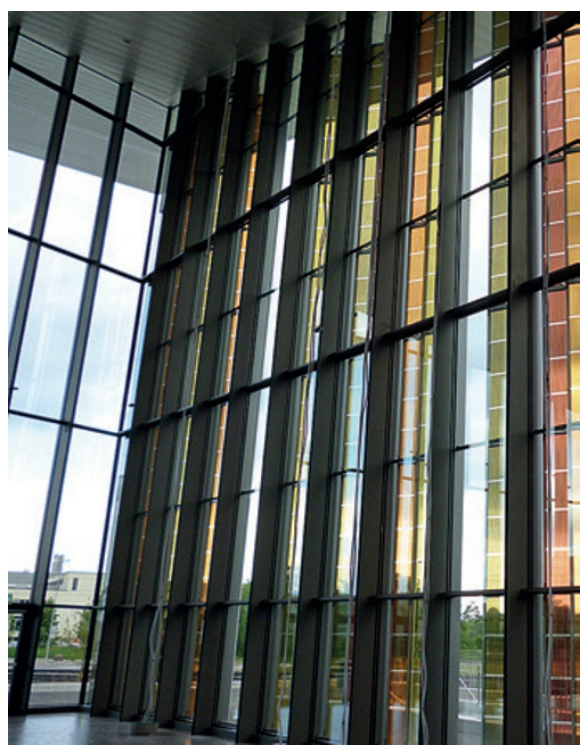

Coloured dye-sensitized solar panels at the Swiss Tech Convention Centre, Lausanne.

highly efficient perovskite-based photovoltaic glazing panels could soon make their market debut.

However, as pointed out by Grätzel, some open issues and pitfalls are now being debated in this community. Among these, the main concern revolves around the accuracy of the reported efficiency values. Specifically, hysteretic behaviour due to the strong dependence of the photocurrent on the voltage-scan conditions seems to be particularly pronounced in some perovskite solar cells ${ }^{5}$. This has led to doubts about some reported power-conversion efficiency values, which may be altered by hysteresis-related artefacts.

Researchers in the field should thus pay extreme attention to the validation of new materials and technologies, in particular at their early stages. Risks related to a lack of rigorous application of standard characterization procedures have been periodically discussed, and clear guidelines exist to avoid common mistakes in evaluating the light-conversion efficiency of any photovoltaic technology $y^{6-8}$. Unusual behaviour that may lead to inaccurate estimations of device performance should be openly discussed, so that the characterization guidelines can be updated accordingly. Importantly, researchers should always follow these protocols once they have been established. This may sound obvious, but it's not: as will be reported in a survey in the September issue of Nature Photonics, about one third of research articles on high-performance organic or hybrid solar cells published in a small selection of journals in 2011 and 2012 may have reported overestimated efficiency data. In the September issue of Nature Nanotechnology, analogous problems in the definition of reliable measurement routines for nanostructured solar cells will be discussed.

Seeking confirmation of photovoltaic performances from independent certification laboratories ${ }^{6,7}$ should be common practice in particular when record efficiency values are claimed. Furthermore, authors, reviewers and editors ought to check before publication that the characterization of materials and devices is exhaustively described. Nature Materials strongly encourages the reporting of additional experimental data that clearly assesses the performance of perovskite solar cells with respect to stability and hysteresis.

The exceptional performance of hybrid perovskite materials may revolutionize the field of renewable energy with cheap solar cells having power-conversion efficiencies comparable to those of silicon photovoltaic devices. To speed up technological development and to reinforce the trust of potential investors, researchers involved in the competition for ever higher efficiencies should be reminded of best-practice characterization procedures to avoid the publication of unrealistic or inaccurate claims and of the need to share all the information needed to properly evaluate their results. As the saying goes, with great power comes great responsibility.

\footnotetext{
References

1. Green, M. A., Ho-Baille, A \& Snaith, H. J. Nature Photon. 8, 506-514 (2014).

2. http://www.nrel.gov/ncpv/images/efficiency_chart.jpg

3. http://www.oxfordpv.com/\#

4. http://actu.epfl.ch/news/epfl-s-campus-has-the-world-s-firstsolar-window/

. Snaith, H. et al. J. Phys. Chem. Lett. 5, 1511-1515 (2014).

6. Snaith, H. Nature Photon. 6, 337-340 (2012).

7. Yang, X., Yanagida, M. \& Han, L. Energy Environ. Sci. 6, 54-66 (2013).

8. Emery, K. in Handbook of Photovoltaic Science and Engineering 2nd edn (eds Luque, A. \& Hegedus, S.) 797-840 (Wiley, 2011).
} 